\title{
Estudio de parámetros metabólicos en sujetos jóvenes sometidos a ejercicio sub-máximo después de la administración de mate de coca, en la exposición aguda de altura
}

Elydia Mujica, Gerardo Ronceros, Javier Torres, Rogelio Pinto, Walter Ponciano, Yelitz Bravo, Milena López, Duber Gallardo, César Salinas, Segundo Calderón, Carmen Villareal, Manuel Yampufé

Instituto Nacional de Biologia Andina. Instituto de Investigaciones Clínicas y Sección Fisiología de la Facultad de Medicina, UNMSM

Objetivos: Determinar los parámetros metabólicos glucosa, colesterol, triglicéridos, HDL, en sujetos jóvenes sometidos a ejercicio sub-máximo después de la administración de mate de coca, en exposición aguda de altura.

Diseño: Estudio experimental.

Institución: Instituto Nacional de Biología Andina. Instituto de Investigaciones Clínicas y Sección Fisiología de la Facultad de Medicina, UNMSM.

Participantes: Estudiantes universitarios varones nativos del nivel del mar.

Intervenciones: Se estudió siete jóvenes voluntarios sanos, del nivel del mar (NM) (Lima, 150 m), edad 22,43 \pm 0,81 años, peso 66,21 $\pm 1,80 \mathrm{~kg}$. Se contó con la firma del consentimiento informado. Antes del viaje se les sometió a un examen clínico y se evaluó los parámetros metabólicos a medir en la altura (ALT). Dentro de las 24 horas de arribo a la altura (Huancayo, $3280 \mathrm{~m}$ ) y en condiciones de ayuno, se les sometió a un ejercicio sub-máximo, utilizando bicicleta estacionaria. Se obtuvo una muestra de sangre para análisis. Luego de un descanso de 30 minutos, en el cual se les dio a beber medio litro de infusión de mate de coca, se sometió a otra prueba de ejercicio sub-máximo, obteniéndose otra muestra de sangre. Los resultados fueron analizados con el paquete estadístico SPSS 19, mediante la pruebas no paramétricas de Friedman y la prueba de Wilcoxon.

Principales medidas de resultados: Glucosa, colesterol, triglicéridos, HDL, en suero.

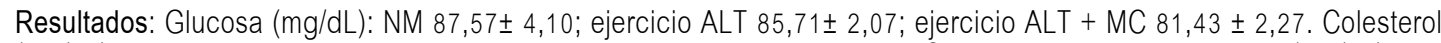
(mg/dL): NM 156,86 \pm 9,14; ejercicio ALT 156,43 \pm 8,78; ejercicio ALT + MC 173,00 \pm 8,76. Triglicéridos (mg/dL): NM

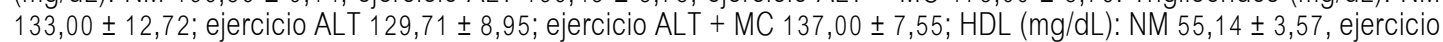
ALT 48,57 \pm 4,85 mg/dL; ejercicio ALT + MC 46,43 $\pm 2,10$. El hematocrito en la altura fue 48,43 $\pm 0,72$.

Conclusiones: En las condiciones experimentales descritas, no se encontró variaciones significativas en los parámetros metabólicos estudiados.

Palabras clave: Parámetros metabólicos, ejercicio, mate de coca, altura. 